\title{
ENHANCED UTILITY BOX USING PELTIER PLATES
}

\author{
P. S. Patil ${ }^{1}$, Adarsh Singh ${ }^{2}$, Parul Patle ${ }^{3}$, Shivani Meshram ${ }^{4}$, Vibha Tembhare ${ }^{5}$ \\ Prashant Gondule ${ }^{6}$, Shubham Ramteke ${ }^{7}$, Rohan ingle ${ }^{8}$ \\ ${ }_{1,2,3,4,5,6,7}$ Department of electrical engineering YCCE, Nagpur,India,441110 \\ ${ }^{18} \mathrm{Head}$ of the Department of electrical engineering, JIT , Nagpur
}

\begin{abstract}
Increase in use of appliances like refrigerator which consists of halocarbons and chlorofluorocarbons, they produces many harmful gas such as Freon which causes green house effect and damage the ozone layer of the earth. Also, the energy consumption is an another concern in today's world. the conventional refrigerator consumes of power ranging from 300 watt to 1500 watt, when calculated for more no of consumers can result in large amount. In this paper advance utility box is used to tackle the above problems. To reduce the emission of harmful gases and global warming, thermoelectric model (TEM) is used. TEM is used for heating and cooling of the system. In TEM, Peltier plates are the main component for heating and cooling purpose. The proposed model is compact in size, lower in cost, and eco-friendly. It is having potential application of storage of food, beverages, medicine at lower temperature and cooling of various electronic devices. This paper will give brief idea about how TEM works and it's several advantages and with the use of this how any model can be developed to the best level of it.
\end{abstract}

Keywords: Peltier effect, Peltier plates, Thermoelectric Module, Peltier refrigeration, Peltier effect.

\section{Introduction}

With the global warming, the release of chloro-flourocarbon (CFCs) in the atmosphere has raised alarm in the nearby days. The major contributors are cooling devices like refrigerator, $\mathrm{ACs}$, etc. The rate of consumption of energy is greater than the rate of generation, At the same time the due the limitations of non-renewable energy sources, it has diverted the research scholars towards the better and more consumption of renewable energy. The use of conventional sources will lead to fatal energy crisis in future and its traces can be seen now also. That's why energy crisis has been labelled as world-wide problem which needs immediate and efficient solutions. To tackle the energy crisis the use of renewable energy sources is increasing day by day. In this paper, solar panel is used to supply power to a utility box which consists of heating and cooling chambers.

With the wide applications of electric energy, and increase in automation, energy demand is also increasing at the same rate. The increase in demand directly affects the conventional devices that consumes more energy such as i.e. refrigerators and heaters, [1] as they consume large amount of power. In addition to this, one of the main disadvantage of the conventional refrigerator is that they are not eco-friendly. Conventional refrigerator contains $\mathrm{CFC}$ as the refrigerant that can cause damage to the environment. CFCs is a synthetic compound of chorine, fluorine and carbon. The typical applications of CFS are in cooling and air conditioners. CFC is the one of the major threat to the environment, it causes depletion ozone layer of the earth's which results into increase in ultraviolet radiation.

The thermoelectric (TE) cooler provides an alternative solution to the above mentioned problems due to its various advantages.

Thermoelectric (TE) cooler

- Do not use ozone depletion such as coolant so it is environment-friendly

- There is no moving part

- Compact in size

- Noise free

- Maintenance free

Along with several advantages it has some limitations like low rating. So, the TEM using Peltier plates is generally used in applications where small size is needed and cooling demand are not too great. The Thermoelectric cooler is used for cooling of electronic devices, storage of medicines, foods and beverages.

\section{A. Thermoelectric module}

A thermoelectric module is a semiconductor based electronic component that function as a small pump moving heat from one side of the device to the other [1]. 


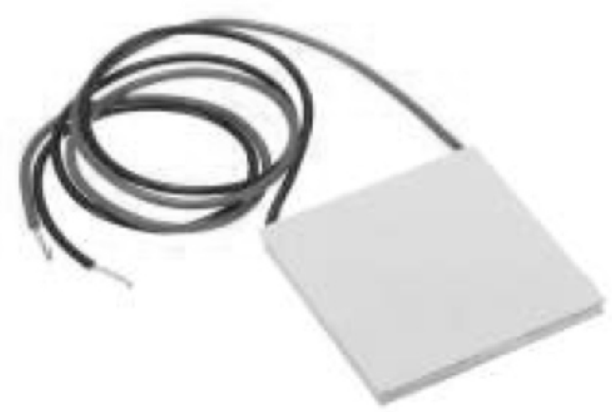

Fig. 1 : Thermoelectric module

A basic thermoelectric module is a semiconductor, consisting of elements (p-type \& n-type). A layer of elements is soldered between two ceramics plates, placed electrically in series structure and thermally in parallel structure[2]. Electrons conducts the heat, by transporting the heat from high state to low state. The transportation capability depends on the depletion layer, it is directly proportional to the number of pairs of ' $n$ ' and ' $p$ ' type (couples). The semiconductor device used in the system is the Peltier plate, which works on Peltier effect. Usually Bismuth Telluride, is the mostly used material to design the Peltier plates and to obtain the Peltier effect

There are various types of Peltier plates available in the market according to the rating. The Peltier Plates used here TEC12706.

Where,

TE - Thermoelectric as it works on thermal and electrical energy.

$\mathrm{C}$ - standard size $(40 \mathrm{x} 40 \mathrm{~mm})$.

In place of $\mathrm{C}, \mathrm{S}$ can also be seen which stands for small size $(30 \times 30 \mathrm{~mm})$

127 - no. of p-n couples.

06 - The maximum current, which is in amperes.

But the major disadvantage of TEM is the low efficiency. So, the TEM using Peltier plates is generally used in applications where small size is needed and cooling demand are not too great.

Thermoelectric module with Peltier plates has been previously used to develop another prototype model [3].

The objective of the paper is to design and present a model which can help to full fill the basic need of cooling and heating at a smaller level and to open a new door for various research work and modification which can increase the efficiency.

\section{Theory}

Thermoelectric Module works on thermoelectric effect. The thermoelectric effect is the transformation of potential difference to temperature difference and vice versa temperature difference to potential difference. A thermoelectric appliance creates potential difference when there is difference in temperature on each side of the plate. On the contrary, when a potential difference is applied across the device, it creates a temperature difference. Thus, an applied temperature gradient causes the charge carrier i.e. electrons in the material to diffuse from one side to another side i.e. form the higher temperature side to the lower temperature side.

The word "Thermo electric effect" can be categorised and explained in following three separately identities [4]

1) Seebeck Effect

2) Peltier Effect

3) Thomson Effect

These effects are explained below :

\section{a. The Seebeck Effect}

Thomas seebeck discovered in 1821 when we make a circuit with junction of two dissimilar metal wire and create a temperature difference at both junction then an electromotive force or potential difference can be achieved that was named as seebeck effect [5]

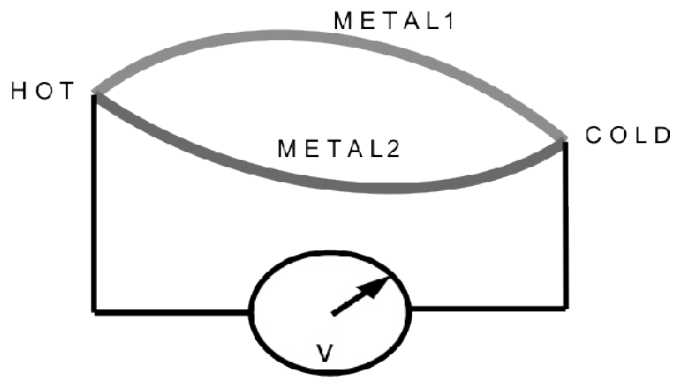

Fig. 2 : Seebeck effect

Whenever there is a difference in temperature across the two sides of a thermoelectric material, holes and electrons move from one side to another, due to movement of holes and electrons an electric Current is induced in the semiconductor materials. The effect that causes this phenomenon is called the Seebeck effect [5]

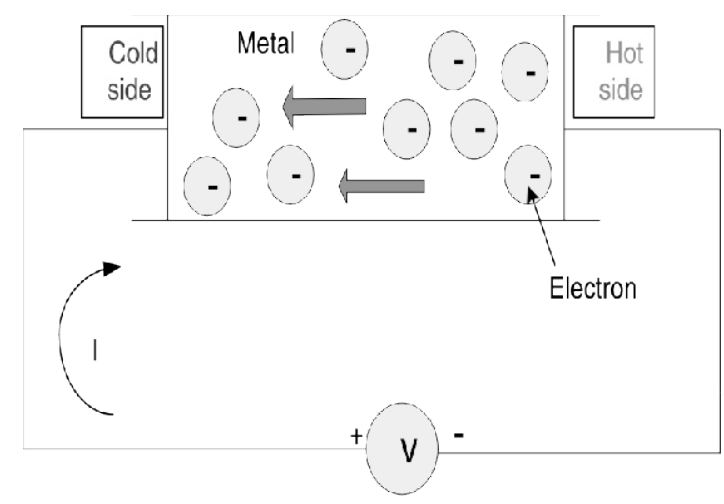

Fig. 3 : Movement of electron in thermoelectric material 


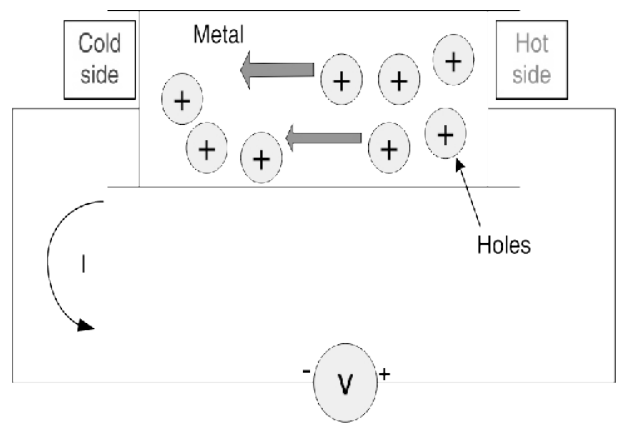

Fig. 4 : Movement of holes in thermoelectric material

\section{b. Peltier effect}

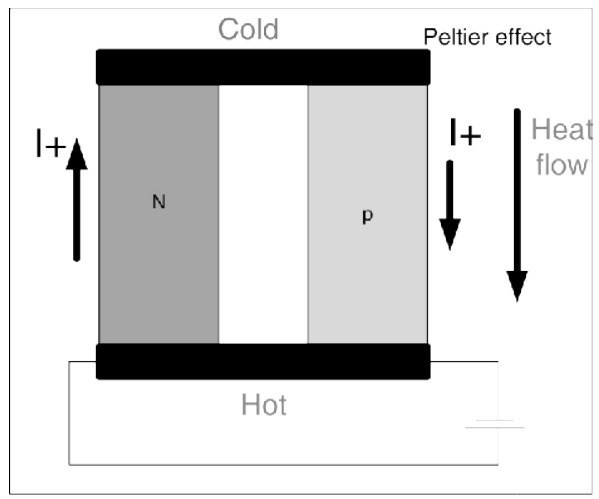

Fig. 5 : Peltier effect

After Thirteen years of Seebeck's discovery, French physicist Jean Peltier found out that the Seebeck effect is a reversible process, or it can be stated that when there is a potential difference between the two sides of the thermoelectric module current flows through the circuit causing one side of junction to be hot and another will become cool.

When there is a current passing through conductor heat is generated, in order to keep the junction temperatures constant heat must be continuously added to or rejected from the conductor.

The total amount of heat added or rejected is directly proportional to the potential difference between the two sides i.e. it is directly proportional to amount of current flowing. This phenomenon is known as Peltier effect which can be expressed as follows [6]

$$
\text { Qpeltier }=\square \mathrm{AB}
$$

Where,

\section{$\square \mathrm{AB}=$ Peltier Coefficient}

Qpeltier $=$ Amount of heat added or rejected from the system

$I=$ Current passing through the thermoelectric material

\section{c. Thomson effect}

In many ways Thomson effect is similar to the Peltier effect, but the main difference is that Thomson effect needs a temperature difference and a flowing current[2]. When the current flows through a wire with a temperature variance, heat will be absorbed or liberated across the wire depending on the material and current direction. Heat absorption or liberation is proportional to the current and the temperature variance. The Thomson heat transfer rate is defined as [6]

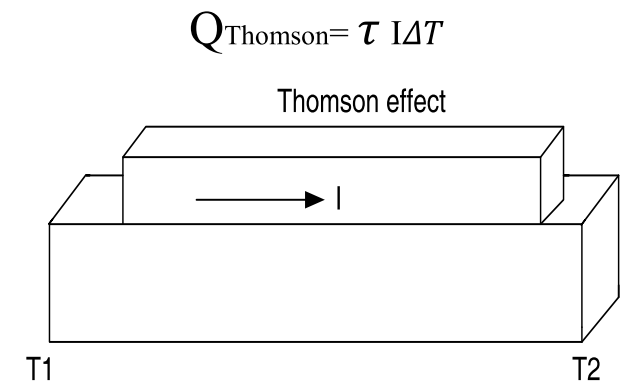

Fig. 6 : Thomson effect

Where,

$\square$ is the Thomson coefficient

\section{d. Photovoltaic effect}

Solar energy is available in abandoned across the earth surface and fuel for conventional sources are depleting day by day. Long back it has attracted the researchers to use solar energy in place of conventional energy sources. In 1839 , French physicist A.E. Bequerel was successful to do so. He discovered the photovoltaic effect. In his experimentation, he explained the production of an electric current with the help of two plates of platinum or gold immersed in an acid, neutral alkaline solution. When this setup is exposed in an uneven way to solar radiation it produces the potential difference at the two plates [7]. The solar panel is used to convert solar energy into electrical energy by means of photovoltaic effect Photovoltaic effect means the conversion of light or radiation into electricity.

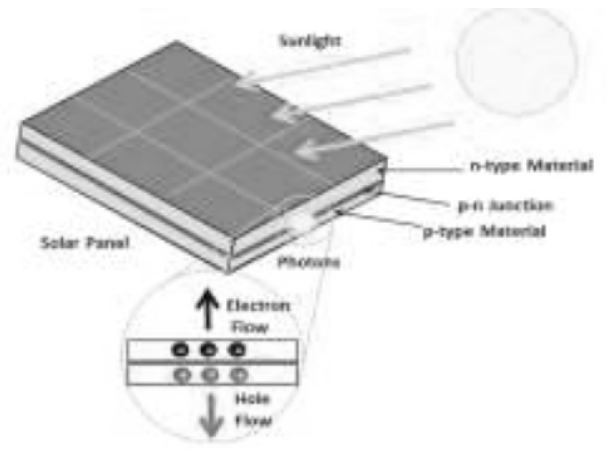

Fig. 7 : Photovoltaic effect 


\section{Experimental setup}

\subsection{Block diagram}

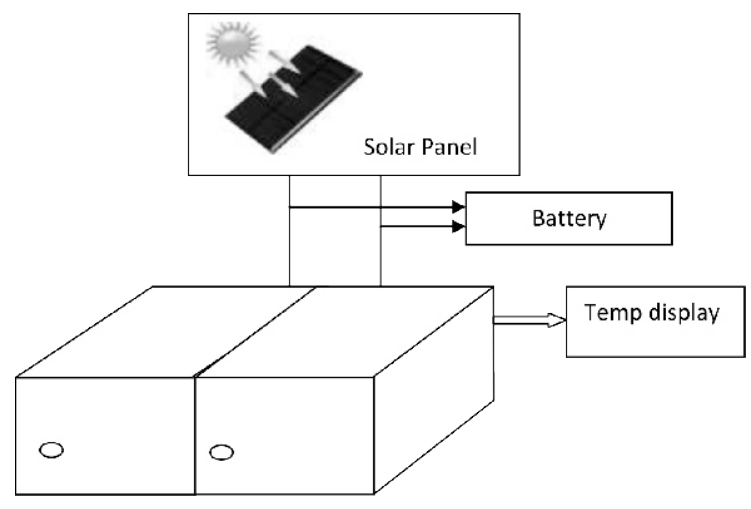

Fig. 8 : Block Daigram

As already discussed TEM is a semiconductor electronic component that functions similar to a small heat pump which moves heat from one side of the device to another. Generally a practical TEM consists of two or more elements of N-type or P-type doped semiconductor material that are electrically connected in series and thermally in parallel [8]. The semiconductor device used here is the Peltier Plate. Here are six such Peltier plates are used. The TEM separates the box into two sections or compartments- one for cooling and other for heating purpose. So, it will be able to provide the temperature over the whole compartment and to facilitate improved results, a heat exchanger is required. To dissipate the heat a passive heat exchanger is used. Heat sink transfers the heat generated by an electronic or a mechanical device to a medium (air, fluid) where heat is dissipated away from the device with the help of exhaust fan, thereby allowing regulation of device's temperature at optimal levels. Heat sink is designed to maximize its surface area in contact with the cooling medium surrounding it such as air. If there is no air flow around the heat sink, energy in the form of heat will be trapped over there causing the burning of the Peltier plates. So along with heat sink, for cooling, convection and transfer of heat, fans are also used. In this model, Axial flow fan are used because of high velocity flow. These fans cause air to flow through it in radial direction through the duct.

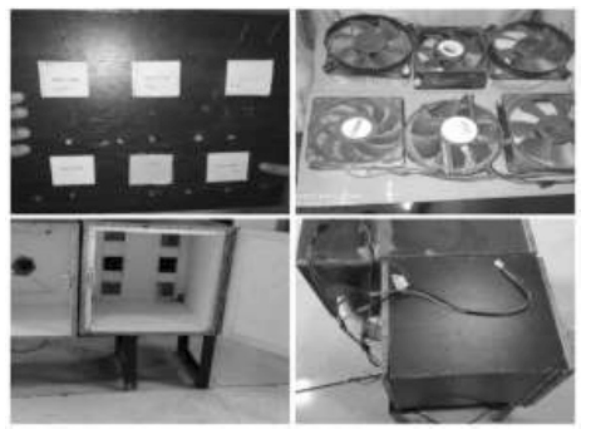

Fig. 9 : Experimental setup for proposed work
The TEM starts working as soon as power supply is ON and the Peltier receives $12 \mathrm{v}$ dc power. So, for power supply, solar panel is used to make the model eco-friendly with renewable source of energy for reduced electricity consumption. But if solar fails or any other problem occur, normal ac power supply from the board can be used. For this, the ac supply is to be converted into $12 \mathrm{~V} \mathrm{dc}$ using complementary source for the Peltier to work.

Temperature sensors with LCD display is used in both the chambers to know the exact temperature. The system needs to be perfectly isolated to get better results. As there are no vibrations, thermal casing is enough to provide insulation and duct tape is used to completely seal this.

The six Peltier plates are connected in parallel and the fans are also connected in parallel. The wire terminals of the Peltier plates and fans are connected to the batteries which are charged through either solar panel or the ac using complimentary source. When the first battery is ON, due to the Peltier effect one side of the Peltier is cooled and other is heated. As far as cooling is concerned, when the battery is switched on the whole cooling chamber will observe a significant change in the temperature, the temperature falls down which is recorded clearly from the temperature display. In view to maintain constant temperature difference between the junctions, the other side of the Peltier starts heating. The fans and the heat sink reject this heat, so the cooling side's temperature again decreases. The fans are also used to circulate this heat in the heating chamber and an increase in temperature is observed. To verify the results several no. of readings were taken at different room temperature. The result is tabulated below \& also expressed in graphical form [fig 10]

TEM system's performance depends on : [9]

1. Potential difference applied between the two sides of the module.

2. Thermal and electrical conductivities of the material used

3. Resistance present between TE module and the heat sink.

4. Thermal resistivity of the heat sink.

\section{Observation Table and Graph}

\begin{tabular}{|c|c|c|c|c|}
\hline Sr. No. & Time sec & $\begin{array}{c}\text { Room Temp } \\
\text { (T1) }\end{array}$ & $\begin{array}{c}\text { Box Temp } \\
\text { (T2) }\end{array}$ & $\begin{array}{c}\text { Peltier temp } \\
\text { (T3) }\end{array}$ \\
\hline 1 & 60 & 26.1 & 1 & 1 \\
\hline 2 & 120 & 26.6 & 2 & 2 \\
\hline 3 & 180 & 27.1 & 3 & 3 \\
\hline 4 & 240 & 28.3 & 4 & 4 \\
\hline 5 & 300 & 26.5 & 5 & 5 \\
\hline 6 & 360 & 26.3 & 6 & 6 \\
\hline 7 & 420 & 26.3 & 7 & 7 \\
\hline
\end{tabular}




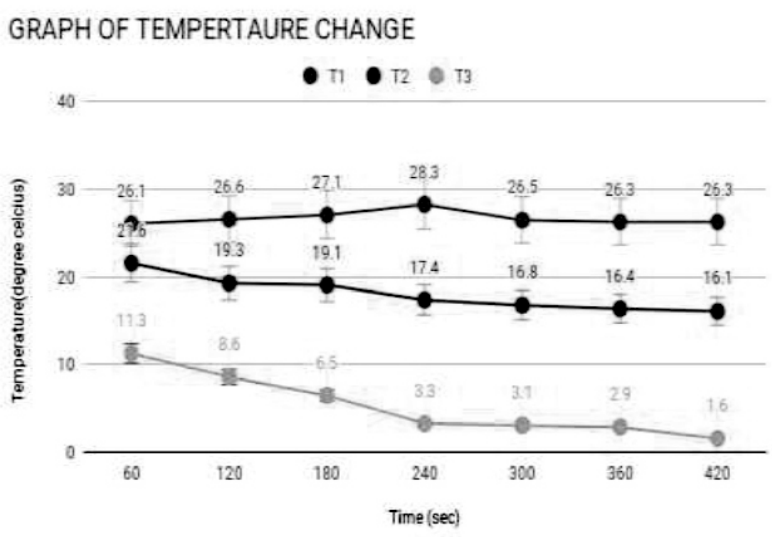

Fig. 10 : Graph plot between time and temperature change

\section{Conclusion}

In this paper a unique application of thermoelectric is discussed. Peltier plates are used for heating and cooling purpose. A portable utility box is designed which can be used for both cooling \& heating purpose. Here Six no's of Thermo-Electric Modules are used. Experimental results show that the utility box designed works properly \& can be used for both heating and cooling purpose. In a timespan of around 30 minutes, the temperature of cooling chamber has dropped from approximate $27^{\circ} \mathrm{C}$ to $15^{\circ} \mathrm{C}$. and there after it remain constant. In this setup external power supply (DC) is applied to the Peltier plates, instead of external power supply renewable sources can be integrated in remote location where power supply is not available. The results may vary with different places and by using better quality Peltier plate The box finds it applications like outdoor tracking, picnic as well as remote locations, as it can be easily carried and used during traveling.

\section{References}

[1] N. Jakhar, N. Baheti, M. C. Gurjar, P. Sharma, "Model development of refrigerator and heater based on peltier module and fresnel lens", Proc. Int. Conf. Recent Advances and Innovations in Engineering (ICRA1E), pp. 1-4, Dec. 2016
[2] H. Lee, "Thermal Design: Heat Sinks, Thermoelectrics, Heat Pipes, Compact Heat Exchangers, and Solar Cells", Hoboken: John Wiley \& Sons, Inc, 2010

[3] A. Jose, A. D'souza, S. Dandekar, J. Karamchandani, P. Kulkarni, "Air conditioner using Peltier module", 2015 International Conference on Technologies for Sustainable Development(ICTSD), pp. 1-4, 2015.

[4] Gupta, K.M., and Nishu Gupta. "Conductive Materials: Electron Theories, Properties and Behavior", Advanced Electrical and Electronics Materials, Wiley 2015.

[5] A. Attar, "Studying the Optimum Design of Automotive Thermoelectric Air Conditioning," Kalamazoo, Western Michigan University 2015

[6] S. Kumar, A. Gupta, G. Yadav and H. P. Singh, "Peltier module for refrigeration and heating using embedded system", Recent Developments in Control, Automation and Power Engineering (RDCAPE- 2015) International Conference, Noida, 2015,pp. 314-319

[7] M. J.Keevers and M.A.Green "Centre for photovoltaic devices and systems" University of New South wales, ,Australia.

[8] Arej Kadhim Abbas, Arshad Hmood Abd Al Kadhim, Haslan Abu Hassan. "Thermoelectric properties and devices of p-type Bi0.4Sb1.6Se2.4Te0.6 and n-type Bi2Se0.6Te2.4 prepared by solid state microwave synthesis", 2012 10th IEEE International Conference on Semiconductor Electronics (ICSE), 2012

[9] Xing cun Colin Tong. "Advanced Materials for Thermal Management of Electronic Packaging", Springer Nature, 2011 